\title{
Improving Learning of Electronic Engineering Skills through e-Learning: a Case Study
}

\author{
Geoff V. Merrett, Alun S. Vaughan \\ Electronics and Computer Science \\ University of Southampton \\ Southampton, UK \\ $\{$ gvm,asv $\} @$ ecs.soton.ac.uk
}

\begin{abstract}
In this paper, we report on changes made to a module that is taken by all first-year electronic engineering students, and which covers transferable and engineering skills. To improve students' perception of and learning experience on the module, half of the taught material was migrated from a traditional 'physical' lecture format to specially recorded 'online' lectures. The changes were evaluated through 1) looking at how regularly students were accessing material, 2) online questionnaires, 3) focus groups, and 4) the standard module evaluation surveys. The changes were very well received by the students and, in a single year, the overall module rating improved from $60 \%$ (static over the preceding three years) to $75 \%$. The changes showed evidence of giving students greater freedom in the way in which they learn, for example allowing them to watch lectures when it best suited their learning style (even if this was at night or on a weekend), pause the lecture while they looked up more information, and rewind sections of the lecture to watch difficult topics again. This has also proved beneficial to international students, as the majority found the online lectures easier to understand, and were able to pause the lecture while they translated unknown words.
\end{abstract}

Keywords—electronics education, e-learning, flip-learning, online lectures,

\section{INTRODUCTION}

Lecture capture is reasonably well established in the educational literature although, more recently, a major focus of this has been on uploading a recording of lectures that the students physically attended (to allow them to later review the material). Examples of this have been reported on UK electronics courses [1], and in other departments at the University of Southampton [2][3]. Such studies have been generally well received by students and educators alike, providing additional learning resources to students, and having minimal impact on physical lecture attendance.

Pre-recorded lectures in place of more traditional physical lectures has also been considered, and can be traced back many decades [4]. It has been suggested that this is a potential method for mitigating problems with the traditional lecture format as a medium for dissemination, where students retain only a small amount of the taught material, do not gain from interaction in large classes, and attendance is not necessarily correlated with performance [5]. Day et al. [6] trialed the provision of pre-recorded lectures (containing audio, video, and slides), and required that the undergraduate students watched these prior to their contact sessions, where they undertook more useful learning activities. When compared to a control group on the same module, the online lectures were shown to increase grades by around $10 \%$. More recently, this method has been termed 'flip-learning' (also dubbed 'Fisch Flip', named after its creator) [7]. Fisch observed the 'traditional' teaching model of delivering lectures to students in class time, and then giving them exercises and activities to undertake independently as homework. He proposed 'flipping' this model, whereby he recorded his lectures for students to watch as homework, and then used the contact time for learning activities where he could be on-hand to help and interact with students.

It is well known that students each have differing and individual approaches, preferences and abilities for learning [8], which are influenced by a broad array of factors including prior experience, age, gender, social background, ethnicity, nationality, language skills and self-perception [9]. However, it is also considered that "learning is most effective when differences in learners' linguistic, cultural and social backgrounds are taken into account" [8]. Overseas students often report linguistic barriers as having a major influence on their academic ability in the UK [10], and a lack of confidence and ability with the language of instruction has been highlighted as a barrier to deeper approaches to learning [11]. Therefore, the ability to pause or rewind an online lecture while an unknown word or phrase is translated has the potential to significantly improve the learning experience for students where English is not their first language.

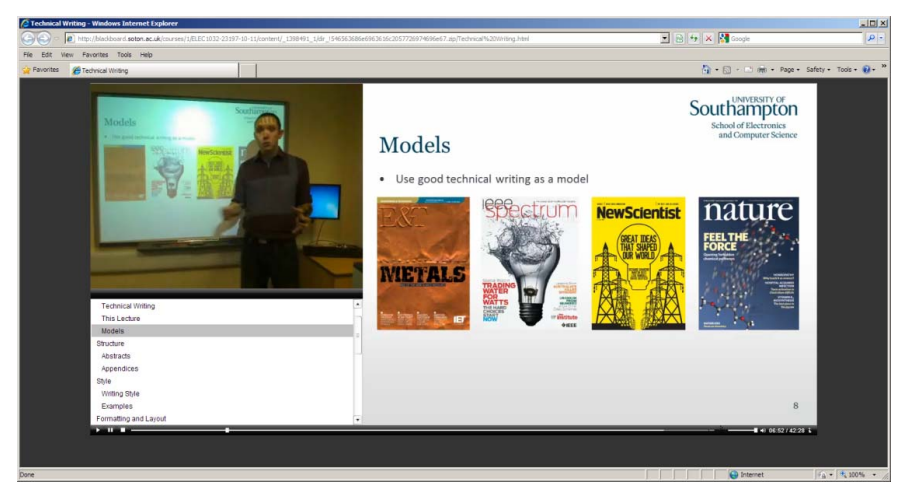

Fig. 1. One of the online lectures in Blackboard, the institutional VLE. 
It has also been reported that students from Confucian cultures, such as China, are mostly taught through 'dissemination' from the teacher to the students, with little discussion or questioning [10]. It may therefore be that many overseas students, particularly when in their first year, will not interact in dissemination-style lectures, instead benefiting more from the advantages of watching and re-watching material online, and subsequently participating in more engaging activities and lectures during contact sessions.

In this paper, we report on changes that were made to a first year module on engineering and transferable skills at the University of Southampton, where material that was noninteractive was moved online in the form of especially videoed online lectures, while interactive sessions (usually containing learning activities or other student-lecturer interaction) remained as physical lectures. The approach was evaluated through a range of means, and the results presented.

\section{Motivation}

Undergraduate degree programmes in Electronics and Computer Science at the University of Southampton take advantage of the research strengths of the department, and provide a rounded education with substantial knowledge and practical experience. They aim to provide students with the upto-date skills required to excel as professional engineers and leaders in business, technology or research.

Engineering Challenges (referred to hereon as 'the module') was a 5 ECTS credit module taken by all 139 firstyear Electronic, Electrical and Electromechanical undergraduates in the department during the academic year that these changes were made and evaluated. The module covers engineering and transferrable skills, e.g. technical writing, academic integrity, experimental design and analysis, laboratory skills and effective library use. Many of these skills are required for later modules, by employers, or to satisfy accreditation requirements. The module also featured a number of 'inspirational lectures', which are given by speakers from both academia and industry.

In preceding years, disengagement had been witnessed as the semester progressed, and the module was poorly received (the overall module rating had remained static at around $60 \%$ over a number of years). Furthermore, attendance at lectures had been noticed to fall significantly after the first few weeks of term. To attempt to resolve these issues, e-Learning was introduced into the module through the delivery of lecture material online. The aims were to:

- improve the quality of students' learning experience (evaluable by gauging student engagement);

- improve students' perception of the module (evaluable through end-of-year module ratings);

- reduce the demand on learning spaces (lecture theatres), by using half of the timetabled lecture slots.

\section{IMPLEMENTATION}

Based on the problems and experiences reported above, it was decided to attempt to integrate pre-recorded 'online' lectures into the module for teaching via the one-way dissemination of information. 'Physical' lectures, however, were retained for learning activities, lecturer-interaction and, to give students opportunities to apply knowledge and theoretical principles obtained via the preceding online lecture(s). While providing the students with the background and information required, a module's learning outcomes are not delivered through teaching but through a student's learning and, more importantly, "what the student does" [18]. Hence, the role of the teacher does not end at creating and uploading an online lecture, but also stimulates discussion on- and off-line, runs and facilitates learning activities, and answer student questions [1].

All of the module's material was categorized into one of two (roughly equally sized) categories: one for background and factual material that could be 'disseminated' to students (to be delivered online), and the other where interaction and learning activities were necessary (to be delivered in a more traditional physical style). In principle, this meant that an unpopular Tuesday, $5 \mathrm{pm}$ timetabled lecture slot could be left unused, with students instead watching the lecture when it best suited them and when it fitted their personal learning styles. In a number of cases, the online lecture was used to teach the theoretical principles, while the subsequent traditional lecture delivered linked and appropriate learning activities, thus best exploiting the contact time. This way of structuring the online/physical lectures was based upon the concept of flip-learning [7].

The uses of lecture capture and e-learning resources have been receiving increasing interest in higher education, both as a way of enriching the student experience and in maximizing the use of staff time. It was anticipated that, by making these changes to this module, students would have greater flexibility in their learning (allowing them to view lectures at times that suited them, and pausing/rewinding sections as required). As a result of this, it was envisaged that students would become more engaged with the material.

The online lectures were delivered using specially recorded videos (supplemented by printable lecture slides and other additional resources) on Blackboard, the institutional Virtual Learning Environment (VLE). A total of 11 online lectures were specially recorded, and given by a number of different academic staff. The majority found the experience somewhat unnerving and were often skeptical of the proposed plan for the module; however, the potential for increased student engagement and more effective use of academic time was attractive and staff were happy to be involved.

The videos were captured using Camtasia Studio, and allowed concurrent viewing of both the lecture slides and audio/video of the lecturer (as shown in Figure 1). The decision was taken to record the lectures in lecture theatres (as if the viewer was sitting in a class) rather than the lecturer recording themselves in their office. This was to make the online lecture as realistic an experience as possible, and hence be a worthy substitute for the physical lecture (but with the additional benefits of anytime access, pause and rewind, etc). It was also decided that capturing a real lecture for use in the following year would not be as effective, as it would then be interspersed with student questions, distractions, and background noise. 


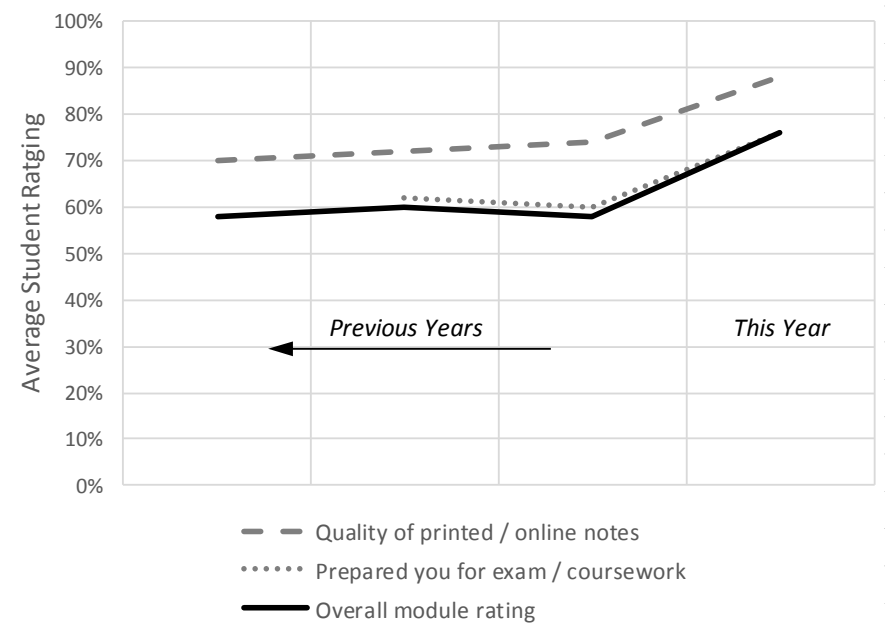

Fig. 2. Average ratings $(100 \%=$ very good, $0 \%=$ very poor $)$ received for the module in the year these changes were made, compared to previous years.

The online lectures were strategically scheduled into the module as if they were physical lectures (delivering the material at the appropriate point in the module), and became available weekly on the VLE at these points. Students were emailed weekly to inform them of this and to ask them to watch the online lecture during the subsequent week. This was done so that they appeared as a coherent part of the module rather than being additional (and optional) resources.

\section{EVALUATION AND RESULTS}

An in-depth evaluation of the changes made was conducted to improve the quality of learning on the module, and to gauge student's perceptions in a UK Higher Education landscape where their financial investment as customers is increasing. To evaluate the students' perception of the online lectures, its impact on quality learning, and the level of engagement with the module material, a variety of methods were employed:

- VLE tracking statistics: to see how regularly, and at which times, students access online material;

- Online survey: to find out student perceptions of the online lectures, conducted through the VLE (completed by 78 students; $55 \%$ of the cohort);

- Focus groups: to obtain more detailed qualitative feedback from a small (but ideally representative) group of 19 students. These were conducted by a postgraduate to encourage open and honest responses;

- 'End-of-module evaluation' forms: to provide insight into perception of the module. These forms asked students to rate various aspects of the module using a 5point Likert scale (converted to percentages in this paper, where $100 \%=$ very good, $0 \%=$ very poor).

These methods allow the evaluation, in detail, of the use and perception of online lectures, plus the students' perceptions of the module as a whole. With the exception of the VLE tracking statistics, opinions were only sampled at a single time (which was during the last few weeks of the module). Ethical approval was obtained from the department's ethics committee.

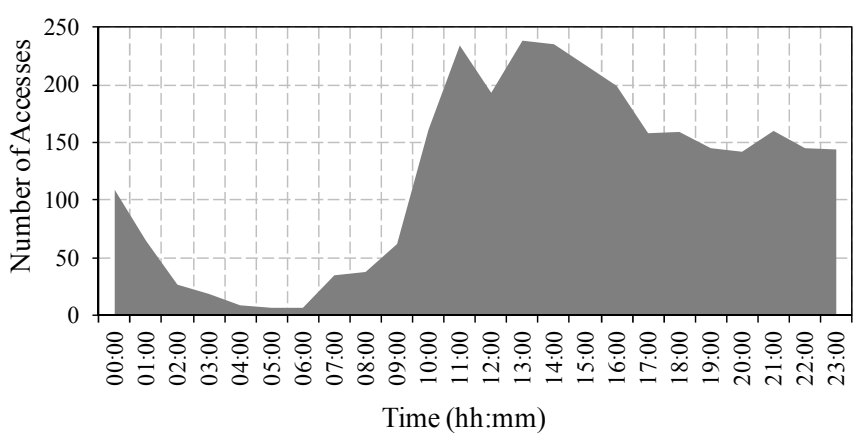

Fig. 3. VLE tracking statistics, showing how students viewed online lectures at different times of the day.

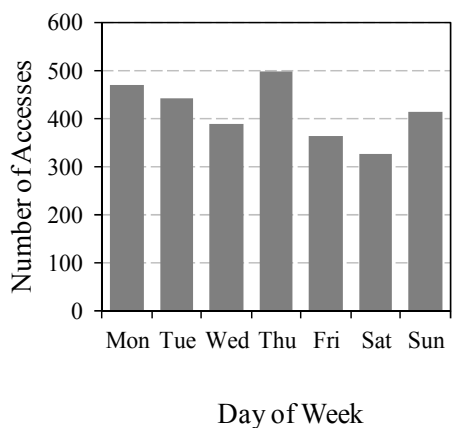

Fig. 4. VLE tracking statistics, showing how students viewed online lectures at different days of the week.

Figure 2 shows the increase in average ratings for the online material, the alignment of the lectures with assessment, and the overall module rating.

Overwhelmingly, over $90 \%$ of students that responded to the online survey were happy with the online lectures, with $49 \%$ preferring them to be delivered online rather than physically, and $43 \%$ not minding. In response to a question on the online survey: "what do you consider to have been the positive aspects of the online lectures, as opposed to attending a physical lecture" (free-text answer), 97\% of responses mentioned one or both of:

- The freedom to watch the lectures when they wanted to, rather than when it was scheduled;

- The ability to re-watch sections (or all) of the lecture.

This is reflected in one student's comment "Can be paused, replayed, done in the morning or at night". These factors also received numerous comments in the focus groups.

VLE tracking statistics for the time-of-day (Figure 3) and day-of-week (Figure 4) that all of the online lectures were accessed show interesting engagement from students. The most popular times to watch the lectures were between $11 \mathrm{am}$ and 4pm; however, a considerable number of accesses also occurred in evenings, and even during the night. Overseas students often adopt different sleeping patterns to their UK colleagues, and the online lectures meant that they could watch these at times when they were most awake and able to learn.

Furthermore, the online lectures were accessed around 750 times at the weekend, with the day of the week having a 
relatively minor effect on the number of accesses. These statistics reinforce the desire and ability of students to learn outside of university hours and timetabled lecture slots. A small selection of the comments received from students relating to this freedom include:

"Freedom to organize when I want to
watch the lecture, that suits me best"

"Can be worked around other

scheduled tasks and deadlines"

"Can access the lecture whenever suits you, at any time of day, and take breaks if necessary without missing any of the lecture. Don't need to spend up to an hour's travel there and back."

"Can watch them in your own time, so that you actually listen to what is being said. In lectures, you tend to dose off"

"I could watch a lecture a week late if I had a difficult coursework assignment, and meant that I definitely 'attended' every lecture"

Furthermore, one student even commented that, as one of the timetabled lecture sessions was on a Tuesday at $5 \mathrm{pm}$, they would have probably missed a lot of them if they were all physical; however, since the majority of the lectures in this slot were put online, they could watch them anytime they liked.

On further inspecting the VLE tracking statistics, it was observed that lectures appropriate to the module's assignments were reviewed again around the submission date. This demonstrates the benefit that online delivery has to students, in enabling them to 're-attend' a lecture again, and schedule lecture 'attendance' around other commitments and deadlines. According to the comments and responses received, the ability to re-watch sections of a lecture was regarded as a significant benefit. This is highlighted in Figure 5, where nearly $90 \%$ of students paused and rewound the videos, while a third watched a lecture multiple times.

The ability to pause and rewind the lecture was particularly well received by a large number of international students, as it enabled them to pause and translate words where necessary. This is reflected in comments including "if unknown terminology appears in the lecture, you can pause, look up the unknown words, and carry on watching!" Additionally, some requested that all of the lectures were also given online in order to allow them to do this for all lectures. As illustrated by Figure $6,40 \%$ of students said that the online lectures were easier to understand, compared to only $16 \%$ which said that it was harder to understand.

A European student commented that it was easier than physically going to lectures, especially as they were not from a culture of asking questions. It is likely that this cultural observation is also mirrored from many Confucian cultures (e.g. Chinese), where students are not encouraged to ask questions or participate in classes; however they are still adopting deep approaches to learning. The delivery of softskills is equally important for international students, as significant differences may exist between the way they were taught in their home countries and the UK (e.g plagiarism).

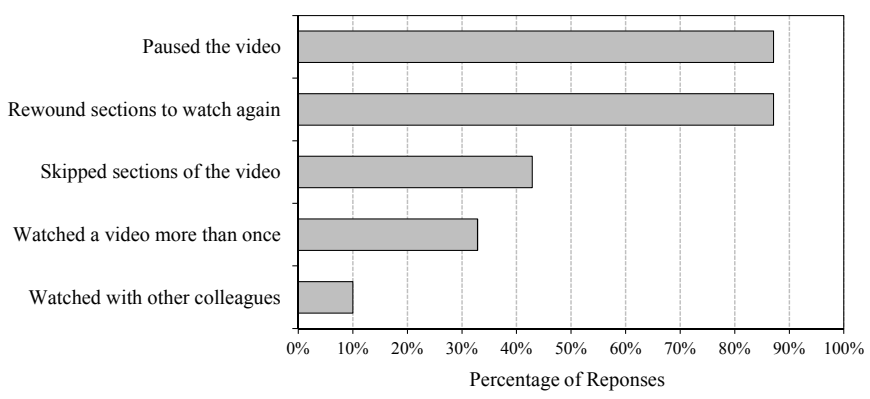

Fig. 5. Responses to the online survey question: "When watching the online sessions, I have... (select all that apply)"

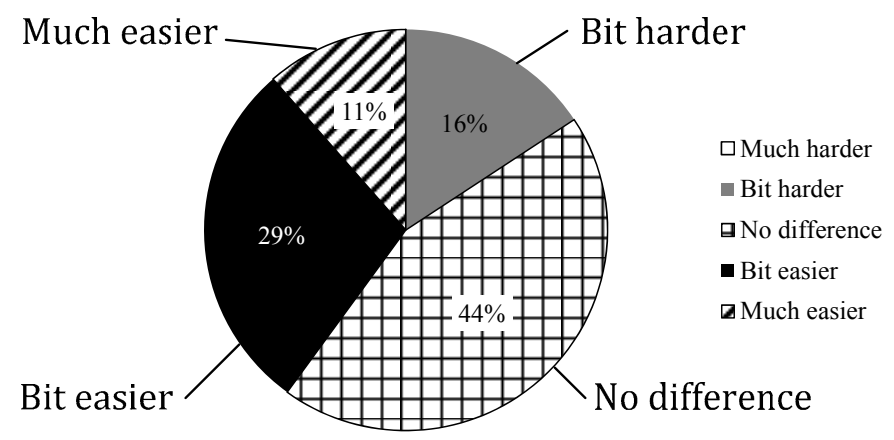

Fig. 6. Reponses to the online survey question: "Compared to physically being in a lecture, was it easier or harder to understand what the lecturer was saying?"

Overall, the online lectures were well received, both in principle (due to the increased freedom and ability to re-watch lectures, discussed above) and in practice (a number of positive comments were received regarding the format of the lectures having slides, plus the video and audio of the lecturer). However, as anticipated, there were negative aspects to the changes. These included a general feeling that the online lectures were less important than physical ones (and hence less important to watch), and students reported motivation/attention problems when watching lectures at home. This is unfortunately inherent in the increased freedom that was very popular, though could potentially be mitigated through, for example, requiring students to complete a short summative test after each video. The predominant negative aspect that students reported in the online survey and focus groups was that they could not ask questions during the online lectures. However, one student did comment in the focus group that:

"You can't ask questions and get a direct answer. But if you don't understand, you can rewind a few times and work it out yourself which, in some ways, could be better for learning"

While we believe that an ability to ask questions of the lecturer is essential in this context, having an element of selflearning (as suggested in above comment) is likely to improve the ability of students to become independent learners.

\section{CONCLUSIONS}

This paper has presented results from an attempt to improve student learning and satisfaction of a first-year electronic 
engineering module through careful use of e-Learning. Results are promising, showing that students engaged with online lectures, and indicated a significant improvement in inclusivity: giving students greater freedom in the way in which they learn. For example, students could watch the lectures when it best suited their learning style (even if this was at night or on a weekend), they could pause the lecture while they looked up more information on a particular term, and they could rewind and watch again difficult topics.

\section{ACKNOWLEDGEMENTS}

Financial support for the evaluation of this work was provided by the UK's National HE STEM Programme (http://www.hestem.ac.uk/).

\section{REFERENCES}

[1] S. Davis, A. Connolly, and E. Linfield, "Lecture capture: making the most of face-to-face learning," Engineering Education, vol. 4, no. 2, pp. 4-13, 2009.

[2] C. J. Andrews, R. C. Brown, C. K. W. Harrison, D. Read, and P. L. Roach, "Lecture capture: early lessons learned and experiences shared," in the Teaching of Physical Sciences, vol. 6, pp. 56-60, Sept. 2010.
[3] J. Copley, "Audio and video podcasts of lectures for campus-based students: production and evaluation of student use," in Innovations in Education and Teaching International, vol. 44, no. 4, pp. 387-399, Nov. 2007

[4] J. F. Gibbons, W. R. Kincheloe and K. S. Down, "Tutored Videotape Instruction: A New Use of Electronics Media in Education," in Science, vol. 195, no. 4283, Mar. 1977.

[5] D. M. Collard, S. P. Girardot, and H. M. Deutsch, "From the Textbook to the Lecture: Improving Prelecture Preparation in Organic Chemistry," in J. Chem. Educ., vol. 79, no. 4, 2002.

[6] J. Day, J. Foley, "Evaluating web lectures: a case study from HCI," in Proc. Computer Human Interaction 2006, Montreal, Canada, Apr. 2006.

[7] D. Pink, The Telegraph (2010, Sept 12), Think Tank: Flip-thinking - the new buzz word sweeping the US [Online]. Available: http://www.telegraph.co.uk/finance/businessclub/7996379/Daniel-PinksThink-Tank-Flip-thinking-the-new-buzz-word-sweeping-the-US.html.

[8] N. Hativa, Teaching for effective learning in Higher Education. Dordrecht: Kluwer Academic, 2000.

[9] H. Fry, S. Ketteridge, and S. Marshall, A Handbook for Teaching and Learning in Higher Education: Enhancing Academic Practice. Abingdon, England: RoutledgeFalmer, 2003.

[10] R. Collins and H. Lim, "Research into the Chinese Students' experience," The Higher Education Academy, 2004.

[11] D. A. Watkins and J. B. Biggs, "Teaching the Chinese Learner: Psychological and Pedagogical Perspectives," Hong Kong, China: Comparative Education Research Centre, 2001, p. 306. 